\title{
Measurement and Analysis of Visual Physical Magnitude of Electroless Copper-Plated Veneers
}

\author{
Sun Lili ${ }^{1, a}$ \\ ${ }^{1}$ College of Art and Design, Harbin University of Commerce, 1 Xuehai Road, Harbin 150028, China \\ a15304658190@163.com
}

\section{Key Words: Wood, Electroless Copper Plating, Color, Glossiness}

\begin{abstract}
The copper-plated veneers made in short process pretreated with $\mathrm{NaBH}_{4}$ are used as the test pieces to measure and analyze the color and glossiness of electroless copper-plated birch and fraxinus mandschurica veneers before and after the coating with the spectral colorimeter and the digital glossmeter so as to provide reference and guidance for the practical design, processing and application of the materials. In the Munsell color system, the colors of the two sorts of copper-plated veneers are respectively marked as 9.8R6.2/9.8 and 9.7R5.9/9.5, with their GZL to be $4.75 \%$ and $3.44 \%$ and GZT $3.35 \%$ and $2.56 \%$, separately. The surface color of the copper-plated veneers is orange red featuring a high degree of saturation and low lightness. After coating, the color tends to be darker red which can arouse the visual psychological perceptions of warmth, luxury and dignity etc; the surface of the veneers boasts unique optical characteristics and copper plating and coating lower the surface glossiness of the veneers and enhance the visual psychological perceptions of reservation, dignity and elegance etc.
\end{abstract}

\section{Introduction}

Wood is the main material composing the interior environment. The wood electromagnetic shielding material made in the electroless copper plating approach is a new composite material applied for decoration and some other functions. It cannot merely maintain the excellent characteristics of wood but also add the color and gloss of copper to the materials, as well as boast higher application value in the interior space requiring electrostatic protection and electromagnetic shielding [1-3].

Color and glossiness are the two major elements composing the characteristics of the visual environmental science of materials and are closely correlated with the comfort degree of people to the visual environment. In the present work, the copper-plated veneers made in short process pretreated with $\mathrm{NaBH}_{4}$ are applied as the test pieces [4-5] and the electromagnetic shielding function of the materials can meet the requirements for civil use and general military use. Measurement and analysis of the surface visual physical magnitude of the veneers before and after copper plating and coating are made to provide basis for the design and application of electroless plated wood composite materials in interior decoration, give guidance and reference for material processing and help enhance the added-value of the materials.

\section{Measuring Methods}

A handheld spectral colorimeter is applied to test four points on both the positive and negative sides of each test piece and each point is tested twice. The mean value of several test pieces under the same conditions is taken to obtain the color parameters of the clad layers before and after the coating (the $L^{*}$ value, $a^{*}$ value and $b^{*}$ value in the 1976CIE $L^{*} a^{*} b^{*}$ color space). $A g^{*}$ and $C^{*}$ are calculated in the formula. There into, $C^{*}$, the degree of saturation of color, is expressed in the formula $C^{*}=\left(a^{* 2}+b^{* 2}\right)^{1 / 2}$ and $A g^{*}$, the hue angle, in $A g^{*}=\arctan \left(b^{*} / a^{*}\right)$. The Munsell color system can directly obtain the measured values of the three attributes of color. The colors have their definite psychophysical meaning but the hue values are not completely digitalized or directly 
measured with instruments. However, the measured values of the chroma index in the $L^{*} a^{*} b^{*}$ color space can be converted into the Munsell color space in the following formula:

$$
\begin{aligned}
& V=0.1002 L^{*}-1.160 \\
& H=-0.03636 L^{*}+0.02663 r-14.30 \theta+0.09131 r \theta+14.826 \\
& C=0.1439 r+1.054 \theta-1.022 \theta^{2}+0.497 r \theta-0.167 \\
& \theta=\arctan \left(a^{*} / b^{*}\right) \quad r=\left(a^{* 2}+b^{* 2}\right)^{1 / 2}
\end{aligned}
$$

In the formula: $V$ is the lightness, $H$ is the hue label value; $C$ is the degree of saturation of color; $\theta$ and $\mathrm{r}$ are respectively the intermediate variables applied in the conversion [6-8].

Measurement is applied with a digital glossmeter under the condition of mirror reflection of $60^{\circ}$. The glossiness refers to the strength of the gloss by means of measuring the reflectivity of the light on the object surface and is expressed with the specific value of the intensity of reflected light and the incident intensity in the formula:

$\mathrm{G}=100 \times \mathrm{R} / \mathrm{R} 1$ 。

In the formula: $R$ refers to the reflectivity of the object; $R_{1}$ represents the reflectivity of the on-gauge plate.

The glossiness value of the wood surface includes the $G Z L$ paralleled with the wood texture, the GZT perpendicular to the wood texture and $G Z B(G Z B=G Z L / G Z T)$ which is the specific value of the two. It is decided by the particularity of the wood texture. Since electroless plating facilitates the wood surface to keep the inherent texture, the measurement of the glossiness of the clad layer still adopts the three indexes.

\section{Test Results}

The colorimetric parametric variation of the surface of the electroless copper-plated veneers is shown in Fig. 1.

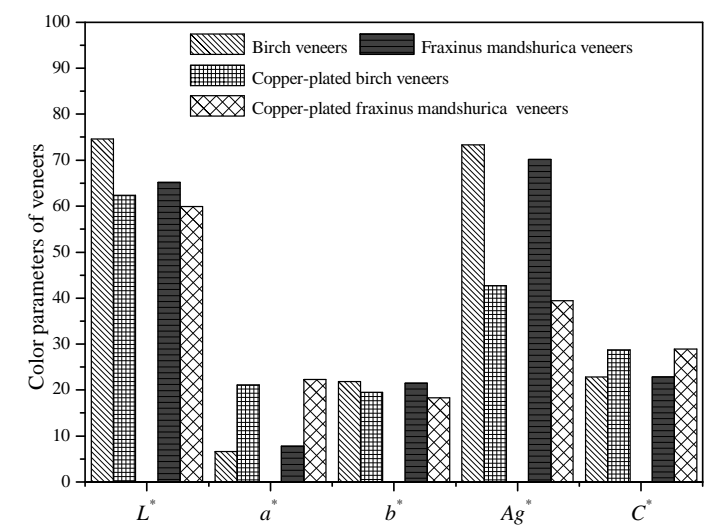

Fig. 1 Comparison of the color parameters of un-plated and plated veneers.

In the distribution of the CIEL ${ }^{*} a^{*} b^{*}$ color space, the value of $a^{*}$ sees the greatest change and those of birch and fraxinus mandschurica are both significantly increased. Then, the value of $L^{*}$ is on the decline. The lightness of the birch veneer is higher and becomes lower after copper plating; that of the fraxinus mandschurica veneer is relatively low and slightly lower after copper plating; the value of $b^{*}$ changes slightly after the plating, indicating minor decrease of the yellow element. Before and after copper plating of the veneers, the value of $\mathrm{Ag}^{*}$ changes greatly and those of birch and fraxinus mandschurica decline sharply. After plating, $C^{*}$, the degree of saturation of veneer color, increases, indicating that the copper-plated veneer turns red and more saturated. 
Table 1. Munsell color parameters of plated veneers and before and after coating.

\begin{tabular}{ccccccc}
\hline $\begin{array}{c}\text { Munsell color } \\
\text { parameters }\end{array}$ & $\begin{array}{c}\text { Birch } \\
\text { veneers }\end{array}$ & $\begin{array}{c}\text { Copper-plated } \\
\text { birch veneers }\end{array}$ & $\begin{array}{c}\text { Copper-plated birch } \\
\text { veneers after coating }\end{array}$ & $\begin{array}{c}\text { Fraxinus } \\
\text { mandschurica } \\
\text { veneers }\end{array}$ & $\begin{array}{c}\text { Copper-plated } \\
\text { fraxinusmandschurica } \\
\text { veneers }\end{array}$ & $\begin{array}{c}\text { Copper-plated fraxinus- } \\
\text { mandschurica veneers } \\
\text { after coating }\end{array}$ \\
$V$ & $9.3 \mathrm{YR}$ & $9.8 \mathrm{R}$ & $9.5 \mathrm{R}$ & $8.8 \mathrm{YR}$ & $6.7 \mathrm{R}$ & 5.9 \\
C & 7.5 & 6.2 & 5.1 & 6.3 & 9.5 & 9.3 \\
\hline
\end{tabular}

Table 1 shows the changes in the Munsell color system, in which the $\mathrm{H}$ indexes (hues) of birch veneer and the fraxinus mandschurica veneer are both in the YR zone near the Y zone. The color of the fraxinus mandschurica veneer more tends to be orange red but slightly dark compared with that of the fraxinus mandschurica veneer. The color marking of the copper-plated birch veneer indicates that the hue label lies in the red zone which approaches the orange zone where $V$ is above the average, $C$ is higher and it is full orange red; the hue label of the plated fraxinus mandschurica veneer shows that its color is basically identical with that of the clad layer of birch, the lightness is of the medium level, the degree of saturation is relatively higher and the color of copper-plated birch veneer is dark red. The color of the electroless copper-plated veneer surface is changed into that of the copper on the basis of maintaining the texture of the material.

Table 2. Glossiness parameters of plated veneers and before and after coating.

\begin{tabular}{|c|c|c|c|c|c|c|}
\hline $\begin{array}{l}\text { Glossiness } \\
\text { parameters }\end{array}$ & $\begin{array}{c}\text { birch } \\
\text { veneers }\end{array}$ & $\begin{array}{l}\text { Copper-plated } \\
\text { birch veneers }\end{array}$ & $\begin{array}{l}\text { Copper-plated birch } \\
\text { veneers after } \\
\text { coating }\end{array}$ & $\begin{array}{c}\text { Fraxinus } \\
\text { mandschurica } \\
\text { veneers }\end{array}$ & $\begin{array}{c}\text { Copper-plated } \\
\text { fraxinusmandschurica } \\
\text { veneers }\end{array}$ & $\begin{array}{c}\text { Copper-plated fraxinus- } \\
\text { mandschurica veneers } \\
\text { after coating }\end{array}$ \\
\hline GZL/\% & 5.33 & 4.75 & 3.52 & 3.52 & 3.35 & 2.68 \\
\hline$G Z T / \%$ & 4.35 & 3.44 & 2.65 & 3.35 & 2.56 & 1.83 \\
\hline$G Z B$ & 1.22 & 1.38 & 1.33 & 1.29 & 1.31 & 1.46 \\
\hline
\end{tabular}

Table 2 shows the results of copper-plated veneer surface gloss measurement. The GZL of birch veneers and fraxinus mandschurica veneers before and after copper plating are higher than the GZT and the $G Z B$ are higher than 1. It shows that the glossiness of the wood texture in the parallel direction is greater than that in the vertical direction. It is the unique optical characteristic caused by the anisotropy of the organization structure of the wood surface and the reason for the differences in color and gloss in different directions when copper-plated veneers are observed. The glossiness of copper-plated birch veneers is higher than that of the fraxinus mandschurica counterparts and is directly correlated with the influence of the surface roughness on the surface appearance and glossiness of the clad layers. As Fig. 2 indicates, $\triangle G Z L$ and $\triangle G Z T$, the difference values of the glossiness of the veneers before and after plating are both negative. It manifests that the glossiness of the materials declines. Due to its low glossiness, the fraxinus mandschurica veneer sees smaller decline than the birch veneer. $\triangle G Z B$ represents the changes of the enhanced glossiness of textures in the parallel and vertical directions. Influenced by the surface texture, the fraxinus mandschurica veneer sees more remarkable enhancement than the birch counterpart.

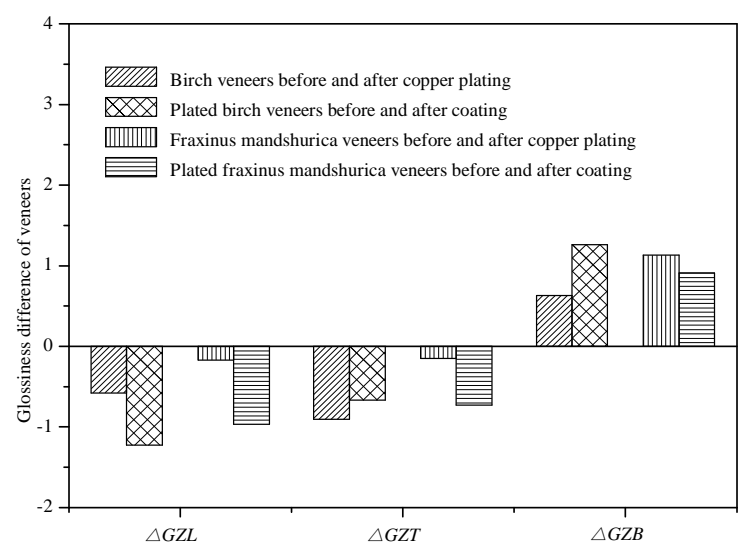

Fig. 2 Comparison of the Glossiness difference of plated veneers and before and after coating 
The glossiness of the veneer surface is influenced by its structure. The cut wood surface is composed of innumerable tiny cell cavities. The glossiness of the inner wall of the cell cavities, the substances in the cavity and the direction of the incident light etc. will exert influence on the refraction and reflection of the light. When the light shoots in the parallel direction of the wood texture, some light will directly reflect on the surface of the dented cell wall after sectioning with the rest of the light reflecting from inside the cell in the direction of long axis of the cell. The scattering degree of the reflected light is low while the mirror reflectivity is high; otherwise, the mirror reflectivity is low. Therefore, the glossiness parallel with the texture is greater than that perpendicular to the texture. The surface texture of the electroless plated veneer is clearly visible with its color changed into that of copper and can be seen in the scanning electron microscope of the clad layer. Since the clad layer is thick, many irregular structure cells are scattered on it on the basis of balanced clad layers on the wood surface and the clad layers display the color of copper. However, the complex interface formed due to the forms and stacking manners of the structure cells also influences the light and develops diffuse reflection, which brings about the soft and unique metallic luster.

Coating can protect the clad layer. In the present work, nitro-lacquer was selected and measurement of the electromagnetic shielding property and visual physical magnitude etc were conducted after the coating and natural airing indoors to study the influence of transparent coating on the color of the clad layer.

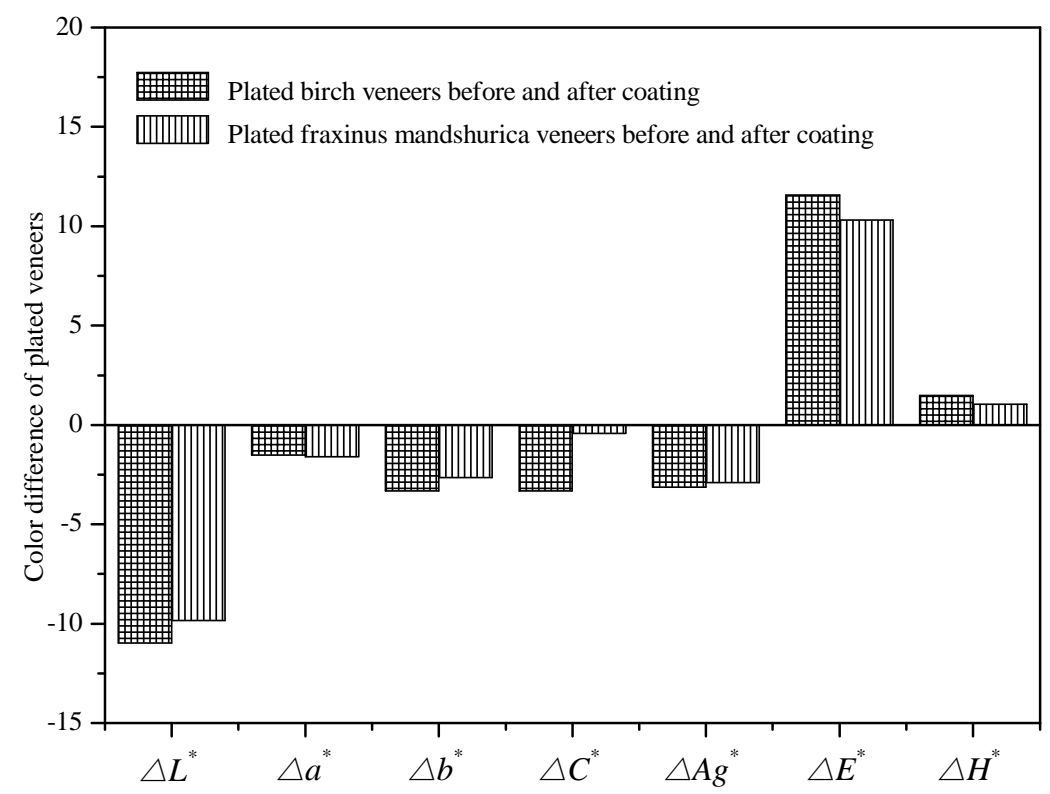

Fig. 3 Comparison of the color difference of plated veneers before and after coating.

Fig. 3 shows the difference values of the color before and after the coating of the copper-plated veneers. $\Delta L^{*}$, which is the lightness difference of electroless copper-plated veneers, is a large negative value. It means that the copper-plated veneers are darker in color and greater in degree after the coating than those before the coating. $\Delta a^{*}$ and $\Delta b^{*}$ are negative values, indicating different degrees of red and yellow after the coating. $\Delta C^{*}$ means the degree of saturation of color lowers and the color gets darker. $\mathrm{Ag}^{*}$ means the hue angle decreases and the color tends to be red on the whole. $\Delta E^{*}$ displays huge change in color difference (between 6 and 12) and the difference before and after the coating can be obviously identified. $\Delta H^{*}$ shows the hue also slightly changes. The analysis tells that the coating significantly changes the color of the veneer surface. The change of color is most influenced by $L^{*}$, followed by $b^{*}$ and $a^{*}$. The labeled values of the colors of the two sorts of veneer surface in Table 1 indicate that their positions in the color cycles after the coating slightly move to the red central zone. $V$ and $C$ also indicate that the copper-plated veneers are slightly darker orange red after the coating, with the results identical with those of the visual test. Table 2 shows the measurement values of the glossiness of the electroless copper-plated veneers before and after the 
coating. The $G Z B$ of birch veneers and Manchurian veneers are still greater than 1 after coating, indicating that the surface of the clad layer after the coating still maintains the unique characteristics of the wood in terms of light reflection; Fig. 2 tells us that $\triangle G Z L$ and $\triangle G Z T$ of the copper-plated veneers before and after the coating are both negative, meaning the glossiness of the veneers lowers after the coating. Different $\triangle G Z B$ values before and after the coating show that the coating exerts some impact on the glossiness of the clad layer. The $\triangle G Z B$ of birch is higher than 1 after the coating. The change manifests that the decrease amplitude of GZL is always greater than GZT. Meanwhile, different $\triangle G Z B$ of two sorts of copper-plated veneers before and after the coating demonstrate that the coating has some influence on the glossiness of the clad layer because the changes occur under the joint effects of the copper-plated layers, veneer surface and paints. During the coating, the original surface structure will be destroyed to different degrees and new surface should be reconstructed, which influences the ratio of the glossiness of the horizontal and vertical textures and leads to different degrees of light reflection of the clad layers.

Electroless copper plating of non-palladium active wood promises excellent electromagnetic shielding properties and unique appearances of the veneers. During its application, its color and glossiness will bring about some visual psychological changes. The evaluation in terms of the visual environmental science based on the influence of the visual physical magnitude on the visual psychological magnitude of the wood in the SD manner shows that red and yellow as the main hues make people feel warm; the redder, the warmer, the emotionally closer to life and sentimental requirements, the more positively associated with the psychological magnitude such as "luxury" and "wealth" and the higher grade of the psychological feeling. The copper boasts high gloss and stylistic, luxury and brilliant visual effects but is easy to generate a sense of psychological distance. At the same time, the materials of high gloss are easy to cause visual fatigue and poor psychological comfort in indoor application. Copper-plated veneers maintain the color of copper, boast the soft light of wood texture, guarantee the visual comfort and display unique advantages of visual environmental science.

The lightness and glossiness of the veneers slightly decline after the coating. The lower the lightness of the material is, the darker and the higher grade it is. Psychologically, the lower lightness it is, the more sense of reservation, dignity and elegance it will generate. The indoor decoration effects show that dark red decoration materials bring a sense of elegance and dignity. Red sandalwood and rosewood, as top-grade furniture materials in China, prove the advantage of dark color decoration wood in the indoor decoration. Meanwhile, dark warm color materials offer the designers more room for color selection and more creative space.

\section{Conclusion}

Electroless copper plating of wood changes the color of the wood surface into that of copper and keeps the original texture features. The color of copper-plated birch and fraxinus mandschurica veneers is orange red featuring a higher degree of saturation and lower lightness in the Munsell color system and is darker red than that before coating; the color of the veneer surface changes greatly and the color change is mostly influenced by $L^{*}$ followed by $b^{*}$ and $a^{*}$; after electroless copper plating and coating, the glossiness declines, the surface of copper-plated wood generates the visual and psychological feelings of "warmth", "luxury" and "dignity" etc and the surface of the materials boasts the unique optical features of wood as well as perfect visual comfort; the coating enhances the visual psychological feelings of "reservation", "dignity" and "elegance".

\section{References}

[1] H. Chohachiro, Y. Kumagai, K. Urabe, Electromagnetic shielding particleboard with Nickel-platedwood particles. J. Porous. Materal, 6 (1999) 247-254.

[2] G. Zhou, G. J. Zhao, Development of Electroless Copper and Gold Plating on wood, Chinese Forest. Sci. Technol. 4(3) (2004) 8-11. 
[3] J. Li, L. J. Wang, H. B. Liu, A new process for preparing conducting wood veneers by electroless nickel plating. Surf. Coat. Technol. 204 (2010) 1200-1205.

[4] L. L. Sun, J. Li, L. J. Wang, Electromagnetic interference shielding material from electroless copper plating on birch veneer. Wood Sci. Technol. 46(6) (2012) 1061-1071.

[5] L. L. Sun, Study on the Preparation of Wood-based Electromagnetic Shielding Material via Novel Electroless Plating Methods. Northeast Forestry University, 2013.

[6] R. M. German, M. M. Guzowski, D. C. Wright, Color and Colorstability as Alloy Design Criteria. J. Met, 32(3) (1980) 20-27.

[7] V. M. Maiorenko, Developing copper alloy with the required color properties. Sov. J. Non-Ferrous Met, 26(8) (1985) 94-97.

[8] H. P. Yu, Y. X. Liu, Z. B. Liu, et al. Prediction of Wood's the environmental Quality by Amendatory Visual Physical Parameters. J. Northeast Forest. University, 32(6) (2004) 39-41. 